\title{
Early-diagnosed silent sinus syndrome and cone-beam computed tomography in a pediatric patient: a case report
}

\author{
Natali Leidens ${ }^{1}$, Ademir Franco ${ }^{2,4}$, Marco C.J. Santos ${ }^{3}$, Irina M. Makeeva ${ }^{2}$, Ângela Fernandes ${ }^{1}$ \\ ${ }^{\prime}$ Department of Stomatology, Federal University of Paraná, Curitiba, Brazil, \\ ${ }^{2}$ Department of Therapeutic Stomatology, Institute of Dentistry, Sechenov University, Moscow, Russia, \\ ${ }^{3}$ Department of Otorhinolaryngology, Paraná State Institute of Otorhinolaryngology (IPO), Curitiba, \\ ${ }^{4}$ Division of Oral Radiology, Faculdade São Leopoldo Mandic, Instituto de Pesquisas São Leopoldo Mandic, Campinas, Brazil
}

\begin{abstract}
J Korean Assoc Oral Maxillofac Surg 2020;46:155-159)
Chronic maxillary atelectasis (CMA) is a progressive alteration in the volume of the maxillary sinuses that may result in facial asymmetry. CMA in asymptomatic patients is known as silent sinus syndrome (SSS) and is a rare entity, especially in pediatric patients. This study reports a case of SSS in a pediatric patient who received an early diagnosis through cone-beam computed tomography (CBCT). An asymptomatic 12-year-old female patient in orthodontic treatment presented with opacification of the left maxillary sinus on a panoramic radiograph. Clinically, the patient had discrete hypoglobus and enophthalmos. CBCT and nasal video-endoscopy revealed ostiomeatal obstruction with bone deformity, leading to diagnosis of SSS. Endonasal endoscopic maxillary sinusotomy was performed. Two years later, the patient remained asymptomatic, and a second CBCT exam confirmed a stable condition. This case highlights the role of optimal radiographic interpretation for early diagnosis of maxillofacial alterations in pediatric patients.
\end{abstract}

Key words: Cone-beam computed tomography, Diagnosis, Maxillary sinus, Atelectasis, Surgery

[paper submitted 2018. 5. 11 / revised 2018. 6. 9 / accepted 2018. 6. 20]

\section{Introduction}

The maxillary sinuses (MS) consist of a pair of pyramidshaped cavities distributed bilaterally in the maxillae ${ }^{1}$. The orbital floor, the alveolar process, and the lateral wall of the nasal cavity and facial surface of the maxilla represent the superior, inferior, and bilateral anatomic limits of the MS, respectively ${ }^{1}$. Communication with the nasal cavity is through an ostium located high in the medial surfaces of these sinus$\mathrm{es}^{1}$. Multiple functions of the MS are reported in the scientific literature, such as mucus production ${ }^{1}$, improvement of voice quality $^{2}$, and participation in maxillofacial growth ${ }^{1}$. Anatomic alterations in the MS, such as ostiomeatal obstruction, lead to

\footnotetext{
Ângela Fernandes

Department of Stomatology, Federal University of Paraná, Av. Lothário Meissner 632, Jardim Botânico, Curitiba 80210-170, Brazil

TEL: +55-41-9-9908-7593 FAX: +55-41-9908-7593

E-mail: angelfnandes@gmail.com

ORCID: https://orcid.org/0000-0001-9019-1522

(c) This is an open-access article distributed under the terms of the Creative Commons Attribution Non-Commercial License (http://creativecommons.org/ licenses/by-nc/4.0/), which permits unrestricted non-commercial use, distribution, and reproduction in any medium, provided the original work is properly cited. Copyright (C) 2020 The Korean Association of Oral and Maxillofacial Surgeons. All rights reserved.
}

functional deficits that have a negative impact on the internal pressure and aeration of the $\mathrm{MS}^{1}$.

Radiographically, ostiomeatal obstruction may be observed in patients as opacification of the $\mathrm{MS}^{3}$. In routine dentistry, several circumstances may lead to sinus opacification ${ }^{3}$. However, in 1964, an unusual clinical entity with sinus opacification and enophthalmos was first reported ${ }^{4}$. Currently, this entity is known as chronic maxillary atelectasis (CMA) and manifests as a decrease in volume of the MS, progressing toward collapse of the sinus walls. This alteration can be classified based on the following severity scale: stage I, membranous deformity; stage II, bone deformity; and stage III, clinical deformity ${ }^{6}$. CMA can be seen in both symptomatic and asymptomatic patients ${ }^{6}$. However, the latter is referred to as silent sinus syndrome (SSS), a subtype of CMA that combines facial asymmetry and lack of nasosinus symptoms ${ }^{6}$. Because patients with SSS are asymptomatic, the disease is often diagnosed late and culminates in inevitable facial asymmetry. Previous studies that explored the etiologies behind CMA reported chronic sinusitis, negative internal pressure, and valvular occlusion by the medial infundibular wall as potential causes ${ }^{5}$. 
In dentistry, the MS are mainly examined with panoramic radiographs and cone-beam computed tomography (CBCT). These imaging modalities are routinely used in orthodontics, stomatology, maxillofacial surgery, and radiology for diagnosis, treatment planning, and follow-up. The detection and optimal interpretation of MS opacification on panoramic radiographs and CBCT scans are important in early diagnosis of SSS, particularly because patients are asymptomatic. Dentists must be aware of the radiographic and clinical signs of SSS to achiever early diagnosis. The present study reports a case of early-diagnosed SSS in an asymptomatic pediatric patient using CBCT.

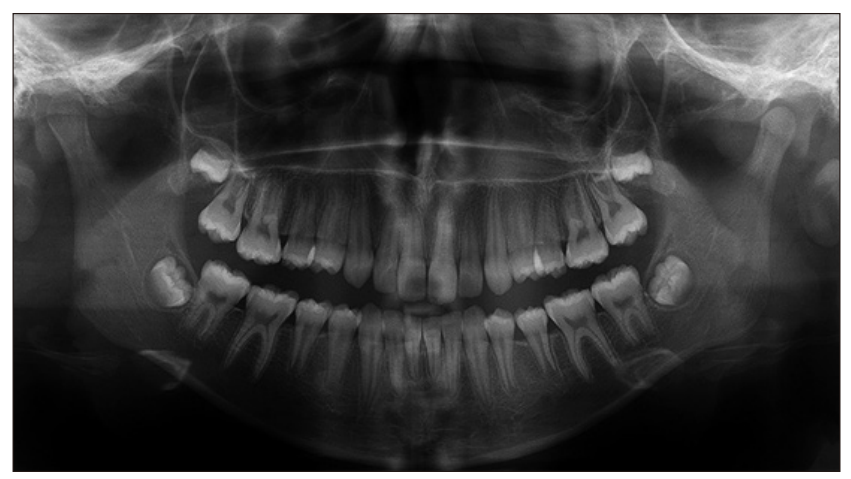

Fig. 1. Panoramic radiograph taken for orthodontic treatment. The panoramic radiograph from November 2015 shows opacification of the left maxillary sinus in an asymptomatic 12-year-old female patient.

Natali Leidens et al: Early-diagnosed silent sinus syndrome and cone-beam computed tomography in a pediatric patient: a case report. J Korean Assoc Oral Maxillofac Surg 2020

\section{Case Report}

In November 2015, a 12-year-old female patient was referred to the local Department of Imaging for analysis of her left MS. Suspicion of a pathological alteration was raised after observing complete opacification of the left MS in a panoramic radiograph previously taken for orthodontic treatment.(Fig. 1)

During the anamnesis, the patient reported no symptoms, no systemic diseases, no previous medical history of therapeutic interventions or trauma in the MS, and no visual field defect. The extraoral physical exam revealed discrete midfacial asymmetry with hypoglobus and enophthalmos.(Fig. 2) The intraoral physical exam showed no infectious or inflammatory conditions in the teeth or soft tissues adjacent to the maxilla.

CBCT scans of the head were performed using an iCAT Next Generation (Imaging Science International, Hatfield, PA, USA) device with the following settings: field of view of $16 \times 13 \mathrm{~cm}$, voxel size of $0.25 \mathrm{~mm}, 37.07 \mathrm{mAs}, 120 \mathrm{kVp}$, and image acquisition for 26.7 seconds. The images were analyzed by an otorhinolaryngologist and a maxillofacial radiologist using the iCat VisionQ (Imaging Science International) software package.

Bone deformity, ostiomeatal obstruction, and opacification of the left MS were observed on panoramic reconstruction of the CBCT scans (Fig. 3) as well as on axial and coronal slices.(Fig. 4) A nasal video-endoscopy performed in December 2015, confirmed the diagnosis of SSS. An endonasal endoscopic maxillary sinusotomy was performed under
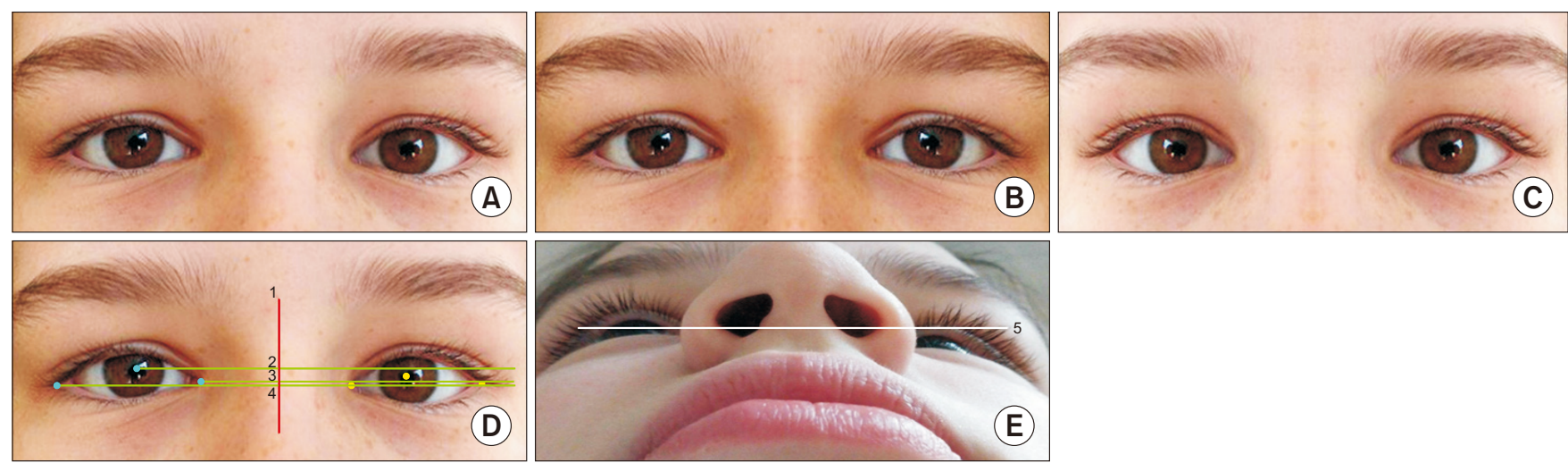

Fig. 2. Discrete facial asymmetry observed in the extraoral exam. A photograph of the patient (A) reveals very discrete hypoglobus on the left side of the face, while more evident differences between the sides are observed with the right (B) and left (C) eyes cropped in a reference midline (' 1 ') or copied and mirrored horizontally. Linear analysis for hypoglobus (D) considering the position of the iris (' 2 '), endocantion (' 3 ') and exocantion ('4') confirmed the discrete vertical asymmetry between the right and left eyes. Enophthalmos (E) was also detected after considering the depth of the orbit (' 5 ').

Natali Leidens et al: Early-diagnosed silent sinus syndrome and cone-beam computed tomography in a pediatric patient: a case report. J Korean Assoc Oral Maxillofac Surg 2020 
general anesthesia by an otorhinolaryngologist using $45^{\circ}$ and $70^{\circ}$ endoscopes to identify the main ostium and enable uncinectomy without injury to the periorbital bone. There were no transoperative complications, and postoperative analgesics and irrigation with saline solution were prescribed. Clearing of the ostiomeatal obstruction was observed and registered throughout 2-year follow-up.(Fig. 5) ${ }^{7}$ The patient remained asymptomatic, and no progression of facial asymmetry was detected during the follow-up period.

\section{Discussion}

SSS is a form of $\mathrm{CMA}^{8}$ that may be incidentally detected in patients with spontaneous midfacial eye asymmetry, such as enophthalmos or hypoglobus ${ }^{8}$. Contrary to patients with CMA, those with SSS do not express nasosinus complaints ${ }^{6}$. Diagnosing CMA or SSS is a challenging task. In fact, most patients are diagnosed late, when facial asymmetries are more

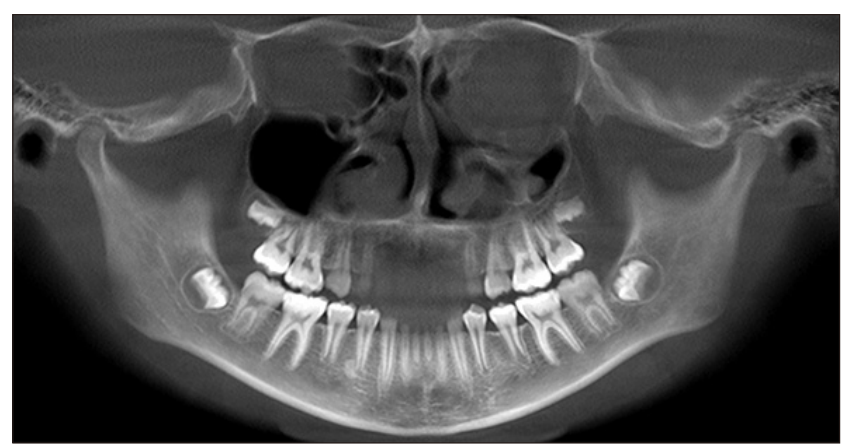

Fig. 3. Panoramic reconstruction of cone-beam computed tomography (CBCT). CBCT panoramic reconstruction from December 2015 shows obstruction of the left maxillary sinus in an asymptomatic 12-year-old female patient.

Natali Leidens et al: Early-diagnosed silent sinus syndrome and cone-beam computed tomography in a pediatric patient: a case report. J Korean Assoc Oral Maxillofac Surg 2020 evident. In this context, it is important to highlight the differential diagnoses of SSS in clinical practice. Clinically, SSS may be overlooked or interpreted as simple facial asymmetry, while radiographically, it may demonstrate signs similar to chronic maxillary sinusitis. The combination of clinical and radiologic evidence must be used to distinguish the disease; the exams must not be evaluated separately. In other words, although SSS may be clinically mistaken for facial asymmetry, the two entities can be distinguished radiographically with the aid of CBCT exams that may show incipient or late involvement of the MS. On the other hand, SSS may be radiographically mistaken for chronic maxillary sinusitis, but the two diseases can be distinguished based on typical clinical symptoms that are reported in chronic sinusitis but missing in $\mathrm{SSS}^{8}$.

Attempting to explain the pathophysiologic process in SSS, the current scientific literature points toward hypoventilation of the MS secondary to obstruction of the ostiomeatal complex, directly affecting the process of gaseous exchange and leading to internal negative pressure ${ }^{9}$. Next, damage in the bony limits of the MS, such as the floor of the orbit, can be induced by gradually increasing pressure, leading to further facial asymmetry ${ }^{9}$. Routine dental imaging can reveal maxillofacial diseases, especially SSS because it is a progressive asymptomatic disease. The present study reported a case of SSS in a 12-year-old female patient who was examined for orthodontic treatment planning with the aid of a panoramic radiograph, allowing early diagnosis of SSS. A systematic review of 55 cases showed that the mean age of patients affected with SSS or CMA was 37.64 \pm 1.48 years $^{8}$.

Detecting alterations in the MS and diagnosing SSS are only possible with imaging. Several imaging modalities were used in the present case, namely panoramic radiograph (bidimensional modality), CBCT (three-dimensional modality),
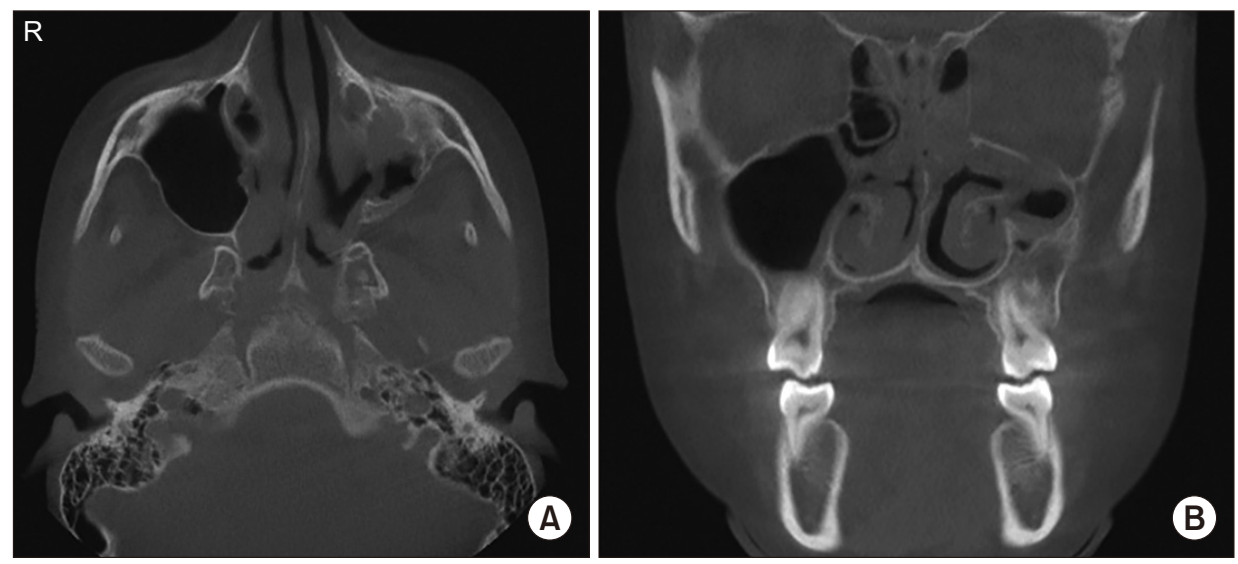

Fig. 4. Axial (A) and coronal (B) slices of cone-beam computed tomography (CBCT). Axial and coronal slices of CBCT from December 2015 confirm ostiomeatal obstruction of the left maxillary sinus in an asymptomatic 12-yearold female patient, leading to the diagnosis of silent sinus syndrome.

Natali Leidens et al: Early-diagnosed silent sinus syndrome and cone-beam computed tomography in a pediatric patient: a case report. J Korean Assoc Oral Maxillofac Surg 2020 

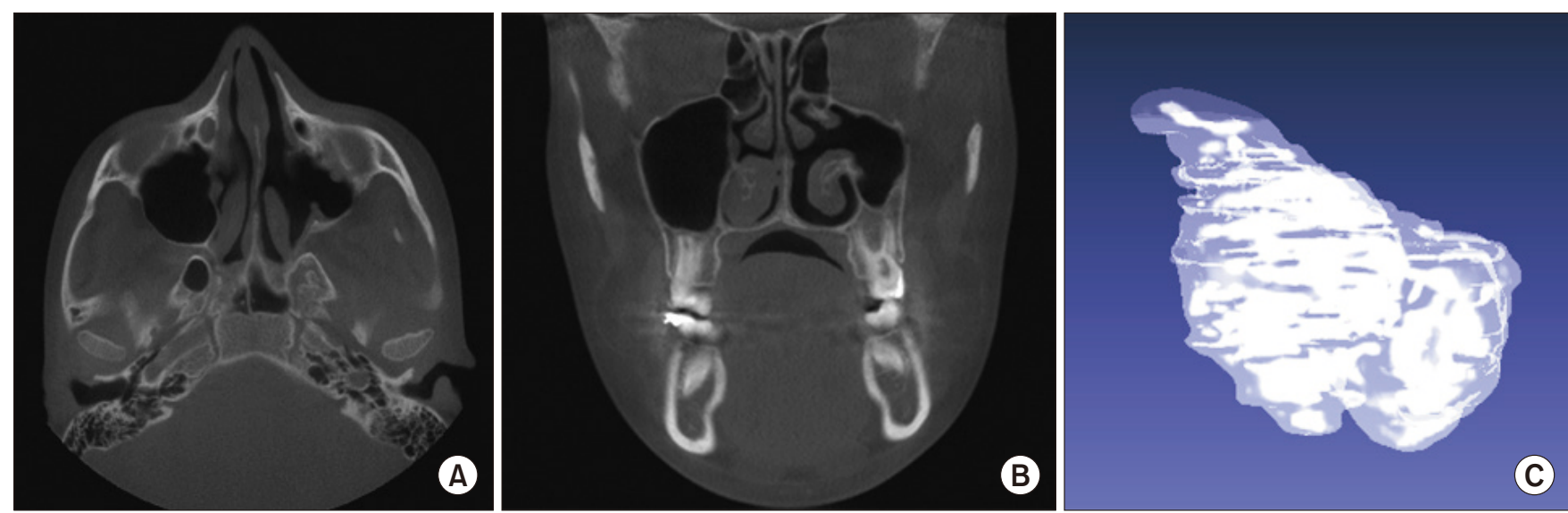

Fig. 5. Axial (A) and coronal (B) slices of cone-beam computed tomography (CBCT) and an approximation of the segmented left maxillary sinus (C). Axial and coronal slices of CBCT from February 2018 during the follow-up period of 2 years. These images demonstrate no ostiomeatal obstruction in the left maxillary sinus after endoscopic endonasal maxillary sinusotomy. The left maxillary sinus was segmented from the CBCT slices and examined using InVesalius ${ }^{7}$ 3.1.1 (CTI Renato Archer, Campinas, Brazil) and MeshLab (ISTI-CNR, Rome, Italy) software packages, respectively.

Natali Leidens et al: Early-diagnosed silent sinus syndrome and cone-beam computed tomography in a pediatric patient: a case report. J Korean Assoc Oral Maxillofac Surg 2020

and nasal video-endoscopy (non-radiographic modality). Each exam played an essential part in the diagnosis of SSS. Panoramic radiographs are consolidated as common imaging exams in routine dentistry. The present study highlights the importance of radiological interpretation in dental practice. Furthermore, analysis of radiographs must not be restricted to the teeth but also must include the maxillofacial bones. Proper knowledge on the interpretation of maxillofacial alterations is also essential. In the present study, the patient was referred to an otorhinolaryngologist and a maxillofacial radiologist who noted not only the radiographic alteration, but also the discrete enophthalmos and hypoglobus. These signs and the lack of symptoms, which had been previously reported in the scientific literature ${ }^{4-6,8}$, led to suspicion of SSS. Thus, threedimensional imaging was used for a more detailed analysis of the sinus morphology ${ }^{10}$ and the inherent alteration of the left $\mathrm{MS}^{11}$. CBCT revealed ostiomeatal obstruction and reduced limits of the left MS. Finally, video-endoscopy confirmed ostiomeatal obstruction finding and led to an endonasal endoscopic maxillary sinusotomy. Computed tomography and endoscopic surgery were previously used for diagnosis and treatment of similar maxillary alterations, which also resulted in positive outcomes ${ }^{6}$.

Case reports of SSS are uncommon, especially in pediatric patients ${ }^{8}$. Additionally, most of the cases in the literature were diagnosed in otorhinolaryngology or ophthalmology. The detection of maxillary alterations on panoramic radiographs is essential for early diagnosis of SSS. Dentists must be trained and aware of their role in prevention of severe sequelae that can result from late diagnosis of SSS. A parallel, multidisciplinary strategy from diagnosis to treatment of SSS must be considered for optimal outcomes.

\section{ORCID}

Natali Leidens, https://orcid.org/0000-0002-6826-7866

Ademir Franco, https://orcid.org/0000-0002-1417-2781

Marco C.J. Santos, https://orcid.org/0000-0003-1807-6081

Irina M. Makeeva, https://orcid.org/0000-0002-1115-6647

Ângela Fernandes, https://orcid.org/0000-0001-9019-1522

\section{Authors' Contributions}

N.L. and A.F. participated in conceptualization, data collection, data analysis, resources, visualization, drafting and final writing. M.C.J.S. and I.M.M. participated in conceptualization, methodology, data analysis, resources, software, drafting and final writing. Â.F. participated in conceptualization, methodology, data analysis, resources, software, supervision, organization, drafting and final writing.

\section{Consent for Publishing Photographs}

Written informed consent was obtained from the patient for publication of this article and accompanying images. 


\section{Conflict of Interest}

No potential conflict of interest relevant to this article was reported.

\section{References}

1. Batista PS, Do Rosário Junior AF, Wichnieski C. [A contribution to the maxillary sinus study]. Rev Port Estomatol Med Dent Cir Maxilofac 2011;52:235-9. Portuguese.

2. Koo SK, Kwon SB, Chon KM, Kim YJ, Kim YJ. The role of the maxillary sinus on the voice. Eur Arch Otorhinolaryngol 2015;272:2347-50.

3. Broderick DF. The opacified paranasal sinus: approach and differential. Appl Radiol 2015;44:9-17.

4. Montgomery WW. Mucocele of the maxillary sinus causing enophthalmos. Eye Ear Nose Throat Mon 1964;43:41-4.

5. Kass ES, Salman S, Rubin PA, Weber AL, Montgomery WW. Chronic maxillary atelectasis. Ann Otol Rhinol Laryngol 1997;106:109-16.

6. Mangussi-Gomes J, Nakanishi M, Chalita MR, Damasco F, De Oliveira CA. Stage II chronic maxillary atelectasis associated with subclinical visual field defect. Int Arch Otorhinolaryngol 2013;17:409-12.

7. de Moraes TF, Amorim PHJ, de Souza Azevedo F, da Silva JVL.
InVesalius: an open-source imaging application. In: Tavares JMRS, Jorge RMN, eds. Computational vision and medical image processing: Vipimage 2011. 1st ed. London: Taylor \& Francis Group; 2011:405-8.

8. Brandt MG, Wright ED. The silent sinus syndrome is a form of chronic maxillary atelectasis: a systematic review of all reported cases. Am J Rhinol 2008;22:68-73.

9. Singh KA, Barber WB 2nd, Codner MA. Silent sinus syndrome: a rare presentation after botulinum toxin injection. Aesthet Surg $\mathrm{J}$ 2011;31:181-3.

10. Lee JW, Yoo JY, Paek SJ, Park WJ, Choi EJ, Choi MG, et al. Correlations between anatomic variations of maxillary sinus ostium and postoperative complication after sinus lifting. J Korean Assoc Oral Maxillofac Surg 2016;42:278-83.

11. Nishihara K, Yoshimine SI, Goto T, Ishihata K, Kume KI, Yoshimura $\mathrm{T}$, et al. Topographic analysis of the maxillary premolars relative to the maxillary sinus and the alveolar bone using cone beam computed tomography. Oral Surg Oral Med Oral Pathol Oral Radiol 2017;123:606-12.

How to cite this article: Leidens N, Franco A, Santos MCJ, Makeeva IM, Fernandes Â. Early-diagnosed silent sinus syndrome and cone-beam computed tomography in a pediatric patient: a case report. J Korean Assoc Oral Maxillofac Surg 2020;46:155-159. https://doi.org/10.5125/jkaoms.2020.46.2.155 